\title{
SARCOIDOSIS WITH CENTRAL NERVOUS SYSTEM INVOLVEMENT: A CASE REPORT
}

\author{
Georgia Andressa de Carvalho e Lima Santos ${ }^{1, *}$, Barbara Manfroi ${ }^{1}$, Tiago Nardi Amaral ${ }^{1}$, Letícia Maria Kolachinski Raposo
} Brandão $0^{1,2}$

1. Faculdade de Medicina São Leopoldo Mandic, Campinas (SP), Brazil. 2. Hospital Vera Cruz Campinas, Campinas (SP), Brazil.

*Corresponding author: georgia_acls@hotmail.com

\section{BACKGROUND}

Sarcoidosis is a granulomatous disease, characterized by noncaseous granulomatous inflammation. Its prevalence ranges from 0.04 to 64 cases per 100,000 inhabitants worldwide. The disease presents a predilection for the respiratory system in about $90 \%$ of patients. Involvement of central nervous system occurs in $5-15 \%$ of cases. The antitumor necrosis factor alpha (anti TNF-a) therapy with infliximab, has emerged as a neurosarcoidosis treatment option to refractory and steroid-dependent patients. The following report describes a case of thoracic sarcoidosis with probable neurosarcoidosis that maintained the neurological status after prolonged therapy with corticosteroids and cyclophosphamide, followed by anti TNF-a.

\section{CASE REPORT}

This case report refers to a 50-year-old male who has been undergoing psychiatric treatment for one year due to behavior change, apathy, depressed mood and presented a $20 \mathrm{~kg}$ weight loss in this period. After extensive diagnostic investigation, he was referred to rheumatology with the diagnosis of thoracic sarcoidosis with probable central nervous system (CNS) sarcoidosis involvement. Once subacute infections and neoplastic syndrome were ruled out, the following complementary tests are worthwhile being highlighted: chest tomography with extensive mediastinal and bilateral hilar lymph node enlargement; pathological anatomy of mediastinal lymph node with extensive noncaseifying granulomatous process and interstitial lymphoid infiltrate with negative acid resistant fungi and bacilli research; immunohistochemical study with polytypical lymphoid infiltrate with sparse primary lymphoid follicles, trapped amid exuberant granulomatous reaction in mediastinal lymph node, without criteria for lymphoma; magnetic resonance imaging of the skull with retractable lesion with subcortical cortical degeneration of the anterior poles of the frontal lobes, in a bilateral and symmetrical manner, associated with significant local volume loss; angiotensin-converting enzyme, ionic calcium and 1.25 dihydroxyvitamin $\mathrm{D}$ within the normal range. The patient undergone pulse therapy with methylprednisolone, followed by oral corticosteroid therapy and monthly cyclophosphamide for 12 months, with significant regression of mediastinal lymph node enlargement, slight behavioral improvement and no change or progression of the CNS image findings. As the cognitive performance was not as expected, the patient was submitted to infliximab treatment, remaining stable, with no clinical or image changes after 3 months of treatment.

\section{CONCLUSION}

This case report describes a probable neurosarcoidosis, defined as biopsy-confirmed sarcoidosis in an organ outside the nervous system and a neurologic syndrome consistent with granulomatous inflammation. Despite undergoing promising therapies, it is possible that the cognitive outcome did not have much improvement once the cognitive decline was attributed to a psychiatric condition for a long period of time. 